\title{
Galileo Galilei, la perspectiva y la descentración de la Tierra
}

\author{
ANÍBAL SZAPIRO \\ Universidad de Buenos Aires \\ Consejo Nacional de Investigaciones Cientificas y Técnicas
}

Resumen: en este artículo exploro el vínculo entre (i) la original respuesta dada en 1624 por Galileo Galilei a un antiguo argumento contra la descentración de la Tierra y (ii) las premisas para la abstracción y la representación del espacio desarrolladas en el ámbito de las artes mecánicas debido al desarrollo de la perspectiva en la pintura renacentista. En continuidad con las investigaciones de otros, indico los motivos conceptuales, históricos y biográficos que permiten sostener que dicha respuesta de Galileo fue deudora de tales premisas. Finalmente, extraigo conclusiones sobre las relaciones entre las artes mecánicas y las ciencias en el contexto de la denominada revolución científica en contra de quienes niegan la influencia de las primeras sobre las segundas.

Palabras clave: abstracción del espacio, revolución científica, artes mecánicas, observación astronómica. 


\title{
Galileo Galilei, Perspective and Decentration of the Earth
}

\begin{abstract}
In this article I explore the relations between (i) the original response given in 1624 by Galileo Galilei to an ancient argument against the decentration of the Earth and (ii) the premises for the abstraction and representation of the space developed within the mechanical arts due to the development of the perspective in the Renaissance painting. In continuity with others' researches, I indicate the conceptual, historical and biographical reasons that allow us to support that Galileo's answer was indebted to such premises. Thus, I draw conclusions about the relationships between the mechanical arts and the sciences in the context of the so-called Scientific Revolution against those who deny the influence of the former on the latter.
\end{abstract}

Key-words: abstraction of the space; scientific revolution; mechanical arts; astronomical observation.

\section{Introducción}

- $\mathrm{n}$ un trabajo publicado hace algo más de una década en la Re¿ista Latinoamericana de Filosofía, Vidal y Burucúa concluían que Giordano Bruno, en su De Inmenso et Innumerabilis (de 1591), parecía haber "integrado los datos de la visibilidad y de la representación perspectiva entre los elementos probatorios más poderosos de la infinitud del universo" (Vidal y Burucúa 2004: 124). En un trabajo anterior que reflejaba sus investigaciones doctorales, el mismo Burucúa había mostrado que la noción pictórica de perspectiva se encontraba dentro del horizonte de significados que asumía el término "perspectiva" en diversos tramos de la obra de Galileo Galilei (Burucúa 1984). En un trabajo reciente publicado en el Journal for the History of Astronomy, mostré que Galileo Galilei respondió en 1624 de manera novedosa al único argumento matemático-astronómico que, desde la Antigüedad, había sido utilizado para probar la centralidad de la Tierra y cuya formulación más completa había sido desarrollada por Claudio Ptolomeo en el siglo II (Szapiro 2018). En este trabajo sostendré que la respuesta de Galileo al argumento ptolemaico es deudora de su posibilidad de percibir el espacio de acuerdo con premisas desarrolladas por la perspectiva que, según acreditó Burucúa, él conocía y que manifiestan ciertas semejanzas con las que, según Vidal y Burucúa, habían funcionado como sustento para probar la infinitud del universo por parte de Bruno algunas décadas antes.

Para ello, organizo el cuerpo central del trabajo dos secciones. En la sección 1, realizo una breve exposición de las características novedosas de 
la respuesta galileana al argumento matemático contra una posición descentrada de la Tierra. En la sección 2, presento algunas características de la innovación en la abstracción y representación del espacio que supuso el desarrollo de la perspectiva en el Renacimiento y trazo un puente entre ellas y la reinterpretación astronómica galileana que me conducen a suscribir la tesis de una influencia de las artes mecánicas sobre ideas cosmológicas desarrolladas en el siglo XVII, sobre lo que me expido en las conclusiones.

\section{Argumentos matemáticos sobre la posición de la Tierra}

Desde la Antigüedad y a lo largo de la Edad Media se desarrollaron distintos argumentos físicos, teológicos y matemáticos (o astronómicos), tendientes a probar tanto la inmovilidad como la centralidad de la Tierra. Durante la Modernidad, como consecuencia de la postulación por parte de Copérnico de un sistema planetario del que se derivaba que la Tierra poseyera distintos movimientos y que, por tanto, no estuviera en el centro del universo, dichos argumentos fueron sometidos a debate.

La historiografia de la ciencia suele enfatizar el papel del desarrollo de la física inercial en el siglo XVII como medio para socavar los argumentos físicos contra el movimiento y la descentración terrestres. Adicionalmente, se suele señalar al debilitamiento relativo de ciertas posiciones sostenidas por la Iglesia como condición de posibilidad para que el heliocentrismo pudiera sobreponerse a los argumentos teológicos a favor de un universo geocéntrico y geoestático. Los argumentos matemáticos, por su parte, han recibido menor atención. Un posible motivo de tal actitud es que el argumento matemático más conocido -la ausencia de medición de paralaje estelar, que funcionaba como argumento contra el movimiento de la Tierra- fue suspendido con un exitoso ad hoc formulado por el propio Copérnico que, sobrepuesto a algunas críticas iniciales, logró prevalecer hasta que finalmente fue confirmado en 1838 cuando el movimiento terrestre llevaba ya largo tiempo fuera de toda discusión. Tal actitud fue posiblemente interpretada como indicativa de una falta de peso relativo de los argumentos matemáticos en el debate moderno; no obstante, y como lo muestran algunas fuentes del período que consideraré aquí, tales argumentos concitaron una fuerte atención en los siglos XVI y XVII.

Es el caso del único argumento matemático contra la descentración de la Tierra que, si bien fue neutralizado con el mismo ad hoc copernicano que respondía a la falta de medición de paralaje estelar, tiene una historia independiente de aquél. En especial, porque la respuesta ofrecida por Copérnico no se constituyó en la solución definitiva al problema; es posible hallar en Galileo el principio de una solución diferente que supuso una novedosa inter- 
pretación de la experiencia observacional completamente novedosa. Con el propósito de establecer la novedad y especificidad de la respuesta de Galileo, presentaré sucintamente las características del argumento matemático a favor de la centralidad de la Tierra y sendas refutaciones, la copernicana y la galileana.

El argumento matemático antiguo tendiente a probar la centralidad de la Tierra tiene distintas formulaciones, siendo quizá la primera de la que tenemos registro la presente en Fenómenos de Euclides (Euclides 2000b: 270). Todas esas formulaciones se encuentran sustentadas por la interpretación de una misma experiencia. El elemento geométrico central para describir dicha experiencia es el círculo máximo. Un círculo máximo de una esfera es aquel que comparte con ella su radio y su centro, dividiéndola por tanto en dos partes iguales o hemisferios. El argumento consiste en señalar la relación que el horizonte, definido como un círculo que pasa por el ojo del observador y que es perpendicular a su vertical (eje cenit-nadir), tiene con distintos círculos máximos de la esfera celeste, como el ecuador o la eclíptica. La experiencia muestra que estos son divididos al medio por los horizontes de los observadores en cualquier latitud o longitud terrestre. Por tanto, el horizonte es, al menos para los sentidos, también un círculo máximo. Puesto que el horizonte es tangente a la esfera terrestre en todos sus puntos y es, a la vez, un círculo máximo, entonces la esfera terrestre: a) es concéntrica con la esfera celeste y b) posee un radio despreciable comparado con el de esta.

En la formulación más completa y difundida durante la Edad Media y principios de la Modernidad, la desarrollada por Claudio Ptolomeo en su Almagesto (Ptolomeo 1984: 41-42), la prueba estaba estructurada en forma de prueba indirecta (o inducción por enumeración completa). Allí se establecía que si la Tierra no se encontraba en el centro de la esfera celeste podía estar solo en tres lugares cualitativamente distintos de ella: sobre su eje y más cerca de uno de sus polos, fuera de su eje y equidistante de sus polos o fuera de su eje y más cerca de uno de sus polos. Cada una de estas posiciones traía aparejadas consecuencias incompatibles con la experiencia como producto de que el horizonte no bisecaría a los distintos círculos máximos de la esfera y a la esfera misma. Entre ellas, y según la dirección de desplazamiento y la posición del observador, que los intervalos entre solsticios y equinoccios no fueran iguales, que ciertos observadores no experimentaran equinoccios, que los intervalos de tiempo salida-culminación y culminación-puesta de una estrella no fueran iguales o que los observadores no vieran todos la mitad del ecuador celeste y de la eclíptica (para una reconstrucción completa de la prueba, véase Szapiro 2018). Como estas consecuencias no ocurrían, cada una de las alternativas era eliminada, siendo posible entonces únicamente la centralidad de una Tierra, cuyo radio debía ser despreciable en comparación con la distancia a las estrellas fijas.

La solución que halló Copérnico a este problema específico fue pos- 
tular una distancia lo suficientemente grande a la esfera de las estrellas fijas que hiciera que la distancia Tierra-Sol tuviera una magnitud despreciable comparada con aquella (Copérnico 1997: 22-23). Ya no se postulaba solamente, como lo hacía la tradición, la despreciabilidad del radio terrestre sino también la del radio de su órbita. Por ello, debe destacarse que el descentramiento copernicano no fue significativo si se lo evalúa en términos de distancias relativas: la distancia del observador copernicano al centro del universo era, comparada con la distancia a sus confines, igual (o incluso menor) que la distancia del observador ptolemaico.

Esto se hace patente, por ejemplo, en el pensamiento de las primeras generaciones de copernicanos, como Thomas Digges. En su obra $A$ perfit description, muchas veces señalada como pionera de la infinitización del universo en el contexto de una obra astronómica, el orbe de estrellas se elevaba infinitamente en altitud esférica, tal como dejó asentado en el grabado que acompañaba su obra (Digges 1983). Si bien existen debates en torno a si tal extensión era física, ${ }^{1}$ parece claro que Digges seguía comprometido con una idea de un par Tierra-Sol en un centro desde el que se elevaba esa infinitud.

Por otra parte, y por fuera de los autores que adoptaron tempranamente el copernicanismo, la prueba matemática antigua de la centralidad seguía vigente. El caso más significativo es quizá el del propio Galileo en su etapa precopernicana. En su Trattato della Sfera ovvero Cosmografía reproducía el antiguo argumento al decir que

[...] si la Tierra se elevase más cerca de la parte del cielo sobre nosotros, no podríamos sino ver menos que la mitad del cielo, y una mayor parte veríamos cuando, por el contrario, la Tierra se dirigiera hacia abajo; lo que repugna totalmente a la experiencia, siendo que la mitad del cielo es por nosotros continuamente vista. De esto podemos estar seguros observando dos estrellas diametralmente opuestas, de las cuales una nazca en el mismo momento en la que la otra se pone. Porque si el arco del cielo aparente, traspuesto de la estrella oriental a la occidental, fuese menor o mayor que medio círculo, cuando la oriental llegase al ocaso, la otra, no habría aun retornado al horizonte o ya lo habría pasado; lo que repugna a las observaciones, las cuales demuestran esto: que para tales estrellas diametralmente opuestas, el orto y el ocaso se hacen alternadamente en el mismo momento de tiempo; lo que es una evidencia cierta de que el arco entre tales estrellas es por encima de ellas igual al arco que está por debajo. (Galilei 1891: 221-22)

${ }^{1}$ Véase Johnson y Larkey 1934 y Koyré 1979. 
El caso se refiere a dos estrellas sobre el círculo del ecuador que son las que, siendo opuestas, sale una al ocultarse la otra. La experiencia señala que el horizonte divide al medio a tal círculo porque al salir una se oculta la otra, y viceversa. Lo anterior lo conducía a Galileo no dudar de la bisección del cielo por parte del horizonte y, por tanto, a afirmar en el apartado destinado a su estudio (Dell'Orizzonte) que

[...] estando la Tierra, como se ha dicho más arriba, en el centro de la esfera celeste, a nosotros, que estamos sobre la superficie de esta Tierra, solo la mitad del cielo se nos aparece y la otra está oculta; así que si imagináramos, que por nuestros ojos se produjera una superficie hasta el extremo del cielo aparente, esta dividirá la esfera celeste en dos partes iguales, pasando por su centro y siendo, en consecuencia, un círculo máximo; el cual, de esta propiedad de dividir y distinguir la parte del cielo aparente de la oculta, se llama horizonte, esto es, divisor [...] los horizontes son infinitos: porque cualquier vuelta que demos sobre la superficie de la Tierra, iremos, por su redondez, a descubrir y perder de vista una parte del cielo y, en consecuencia, a variar el horizonte. No pueden sino solo dos puntos sobre la Tierra tener el mismo horizonte, y estos son los puntos opuestos diametralmente; y nuestras antípodas tienen el mismo horizonte que nosotros siendo a ellos aparente aquella mitad del cielo que a nosotros se nos esconde. (Galilei 1891: 227)

En 1616, Francesco Ingoli le envió a un Galileo ya heliocentrista un compendio de todos los argumentos teológicos, físicos y matemáticos contra la posición y el movimiento de la Tierra del sistema de Copérnico que él conocía; entre ellos, se encontraba el argumento ptolemaico contra una posición no central de la Tierra sustentado justamente en esta misma apariencia de las estrellas que alternadamente salen y se ponen. Galileo respondió hacia 1624 solo a los argumentos físicos y a los matemáticos, explicitando sobre el que nos interesa una posición esencialmente distinta de la que había sostenido en su juventud y de la que habían sostenido Copérnico y los primeros copernicanos. En sus palabras dejaba entrever su asombro sobre el no poco esfuerzo que había demandado incluso a copernicanos advertir la facilísima respuesta a este argumento:

Dices con Ptolomeo: si la Tierra no estuviese en el centro de la esfera de las estrellas, nosotros no podríamos ver siempre la mitad de esa esfera; pero nosotros la vemos; por lo tanto, etc. Que aquello que nosotros vemos es la mitad, y no más o menos, lo pruebas de varios modos, el primero de los cuales es tomado de las observaciones de dos estrellas fijas opuestas, como lo son el ojo de Tauro y el corazón de Escorpio, de las cuales mientras una nace la otra 
se oculta, y ocultándose una la otra nace; argumento [que vuelve] necesario que la parte del cielo sobre la Tierra es igual a aquella que está debajo, y, en consecuencia, cada una, un hemisferio y la Tierra ubicada en su centro ya que tal accidente sucede en todos los horizontes.

El discurso es bello y digno de Ptolomeo, y acoplado con otra suposición, concluye necesariamente [que la Tierra está en el centro], pero negada ella, el argumento resulta nulo.Y verdaderamente me encuentro maravillado que otros astrónomos de gran nombre y seguidores de Copérnico que se fatigaron no poco para resolver esta instancia, no les haya venido a la mente la verdadera y facilísima respuesta, que es negar el otro asunto de Ptolomeo, del cual adquiere fuerza este argumento. Pero nota, Señor Ingoli, que es verdadero que naciendo y ocultándose alternadamente bajo todos los horizontes dos estrellas fijas, requiere por necesidad decir que la Tierra está en el medio de la esfera estrellada siempre que la Tierra esté inmóvil y que el nacimiento y ocultamiento derive del movimiento de conversión de la esfera estrellada; pero si nosotros (como hace Copérnico) hacemos permanecer quieta a la esfera y rotar sobre sí mismo al globo terrestre, ponlo por donde más te plazca, que siempre habrá dos estrellas fijas como se ha dicho, esto es que nacen y se ocultan alternadamente. (Galilei 1933b: 526-27)

Esto es: si, tal como Copérnico había postulado, la distancia de la Tierra al Sol era despreciable y era la Tierra la que rotaba y no la esfera celeste, entonces la Tierra podía encontrarse en cualquier punto del universo y no habría ninguna clase de incompatibilidad entre la experiencia y una Tierra completamente descentrada: el horizonte continuaría dividiendo al medio a la esfera celeste y a sus círculos máximos y dos estrellas opuestas se comportarían como lo hacen.Y para que el entendimiento sea más claro, ofrece un ejemplo:

sea la esfera de las estrellas, cuyo centro es D, y la Tierra A alejada cuanto se quiera de ese centro, y sea el horizonte siguiendo la línea recta $\mathrm{CB}$.

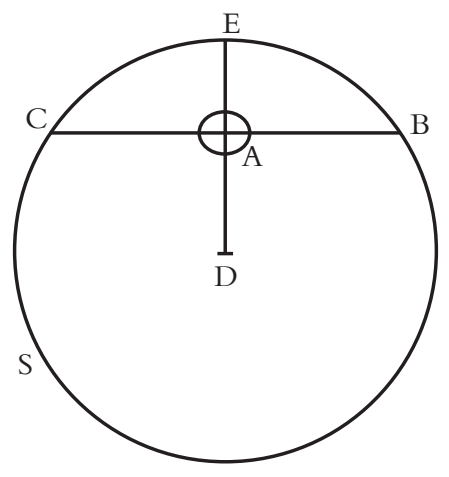


Ahora, si nosotros, estando quietos la Tierra y el horizonte, supusiéramos que la esfera de las estrellas se mueve en torno a su centro D, y una estrella naciera en $\mathrm{C}$, mientras la otra se pone en $\mathrm{B}$, es claro que cuando la $\mathrm{C}$ esté en $\mathrm{B}$, la B no habrá retornado a C (siendo el arco sobre la Tierra CEB menor del que permanece debajo de la Tierra); en cambio estará en S (supuesto el arco BS igual al arco CEB): tardará entonces la estrella B en nacer, después de la puesta de la C, cuanto es el tiempo del arco SC. Pero supongamos ahora que la esfera de las estrellas está fija, y la Tierra móvil sobre sí misma, la cual portará consigo el horizonte $\mathrm{CB}$; y no hay duda alguna de que cuanto el término $\mathrm{B}$ del horizonte estará en $\mathrm{C}$, el otro C estará en B; y donde la primera de las dos estrellas B y C una estaba en el término oriental y la otra en el occidental, hecha tal conversión de la Tierra retornarán en el mismo momento de tiempo invariablemente en los mismos términos; tal que, como ves, ese nacimiento y ocultamiento invariables nada prueban acerca del sitio de la Tierra. Como tampoco de aquello que acrecienta, esto es, que al notarse sobre el círculo vertical siempre 90 grados del cenit al horizonte, se puede inferir que nosotros vemos la mitad del cielo; porque representando en la misma figura la línea BC un horizonte cualquiera, si del centro A se levantara sobre BC una perpendicular que terminara en el punto vertical, ésta contendrá de aquí y de allá dos ángulos rectos, cada uno de los cuales de 90 grados: pues, aquello que son los dos arcos BE, EC, ni se ve, ni se sabe ni se puede saber, ni le sirve a nadie saberlo. (Galilei 1933b: 527-28; figura de Galileo copiada por el autor)

La posibilidad de reinterpretar la experiencia, que otrora repugnaba en palabras del Galileo joven cualquier posición alternativa y que en este pasaje no, requirió de un cambio en los conceptos utilizados para describirla. En la prueba ptolemaica, el horizonte era un círculo producido por la proyección de la visual de un observador sobre la esfera. La esfera era el universo, que tenía un radio determinado que hacía al radio de la Tierra despreciable en comparación con ella. El ecuador era el círculo perpendicular al eje de rotación de la esfera sobre sus polos, y la eclíptica era un círculo máximo oblicuo respecto de este y que determinaba el recorrido del Sol a lo largo del año. Como consecuencia, cuando Ptolomeo desplazaba de manera hipotética a la Tierra, dejaba a todos los elementos de la esfera fijados a su centro. Por ello, los horizontes de una Tierra descentrada no dividirían más a la esfera y a sus círculos máximos a la mitad.

El significado de los conceptos necesitó ser muy distinto en la reinterpretación galileana. En su argumento se puede apreciar ya el paso hacia la definición posterior en el que el horizonte sigue siendo un círculo proyectado por la visual, pero sobre una esfera imaginaria o proyectada con 
centro en el observador y cuyo radio es indeterminado. Los polos pasan a estar determinados por la rotación de la Tierra en torno a su eje con respecto a esa esfera imaginaria (o proyectada), lo que define a su vez al ecuador como el círculo perpendicular a él. La eclíptica es un círculo máximo de esa esfera proyectada, oblicuo respecto del ecuador y determinado por el plano de traslación de la Tierra en torno al Sol. Cuando Galileo imagina a una Tierra fuera del centro del universo, la imagina con la esfera y todas sus partes centradas en el ojo del observador. Por ello, el horizonte continuaría dividiendo a la esfera celeste, a la eclíptica y al ecuador, sin importar su ubicación en el universo. Y dos puntos opuestos en el horizonte, como los de las estrellas que alternadamente salen y se ponen, son opuestos solo debido a la posición del observador, pero ello es independiente de su oposición absoluta.

Un ejemplo que permite esclarecer este punto concierne a otra de las consecuencias que para Ptolomeo debía tener la observación de la esfera desde distintos puntos: una variación de las proporciones de la eclíptica visibles. Mientras para Ptolomeo un observador que no estuviera en el centro no vería seis de los doce signos del zodíaco (sino más o menos, dependiendo de su posición sobre la Tierra y de la cantidad y de la dirección del desplazamiento de esta), para Galileo sí: siempre observaría seis de doce signos sin importar la dirección ni la cantidad de desplazamiento ni la posición del observador sobre la Tierra. Represento esta situación bidimensionalmente en la Figura 1 (pàgina siguiente), en la que la Tierra se ubica en un punto medio entre el centro del universo y su límite. Para Ptolomeo, un observador como el indicado vería cuatro de los doce signos, y uno ubicado en sus antípodas vería ocho. Para Galileo, un observador en cualquier posición sobre esa Tierra vería seis, porque cada una de las partes angulares en las que se divide el zodíaco no está definida sino en virtud de la posición del observador, que siempre observó el universo desde ese mismo lugar.

Copérnico, aun habiendo postulado la rotación y traslación de la Tierra y la despreciabilidad de la distancia al Sol comparada con la distancia a la esfera de las estrellas fijas, evidentemente no alcanzó a advertir plenamente las posibles consecuencias de su sistema y no pudo despojarse del significado de los conceptos de la astronomía de posición, tal como habían sido utilizados por muchos siglos en la práctica astronómica. Galileo, en cambio, pudo dar un paso más en la desvinculación de la apariencia y la realidad. Al asumir a la esfera celeste como una apariencia para cada observador dado y al horizonte como un círculo máximo de esa esfera aparente con centro necesario en el observador, relativizó la totalidad del fenómeno visible al observador quitándole valor probatorio a su centralidad respecto de las apariencias. 


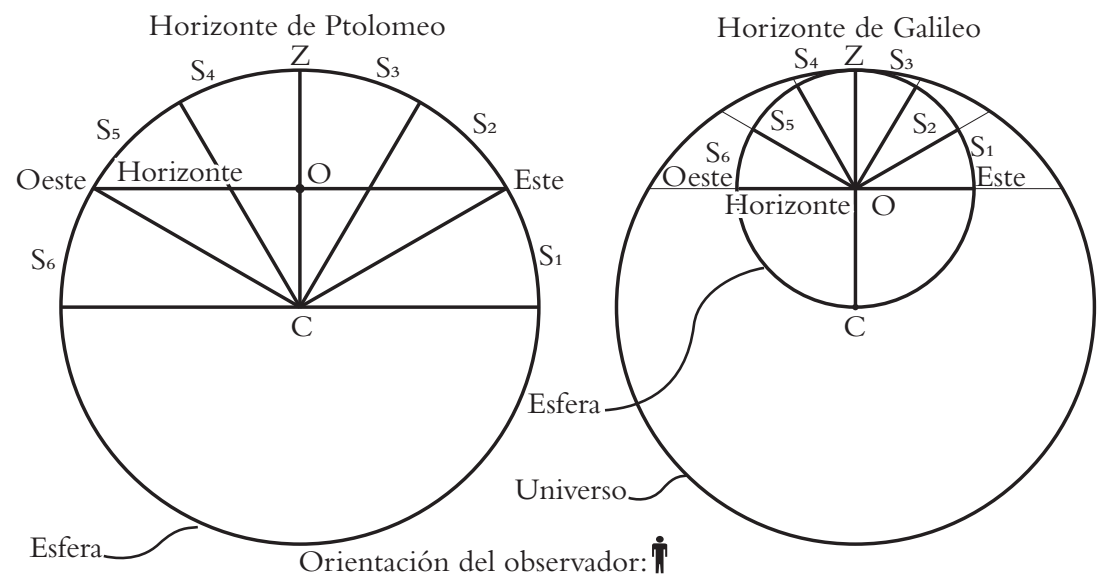

Figura 1

Comparación entre las concepciones ptolemaica y galileana sobre la relación del horizonte con la eclíptica para un observador en una Tierra desplazada hacia su cenit. Nótese que mientras para Ptolomeo lo que rota es la esfera externa, para Galileo lo que rota es el punto en el que se encuentra el observador y, por tanto, el horizonte (por lo que la orientación del observador en un caso es siempre la misma y en el otro, es solo una vez por día). Figura original del autor.

Una pregunta pertinente desde una perspectiva histórica y epistemológica es cuáles fueron las condiciones que permitieron a Galileo una comprensión novedosa de la relación entre apariencia y realidad. El hecho de que Copérnico hallara una solución al problema que no requería un cambio tan dramático en la concepción de la relación entre el observador y el espacio, permite afirmar que no puede ser invocada la teoría copernicana como condición suficiente de ese cambio operado por Galileo. El movimiento de la Tierra no necesitaba, per se, ni la disolución de la identidad entre universo y esfera celeste ni la aceptación de que el fenómeno observado constituía una proyección de un universo cuyos rasgos principales se desconocían. De manera que difícilmente pueda invocarse a la teoría como causa del cambio conceptual.

Las observaciones ocurridas entre 1543 y 1624 tampoco parecen haber sido la clave para el paso hacia este cambio. El telescopio, más que instigarlo, proporcionó evidencia que confirmaba la hipótesis ad hoc copernicana de que la esfera celeste era mucho mayor y, por tanto, el descentramiento terrestre despreciable. Por otra parte, la observación de los cometas y novas posiblemente sí contribuyó a horadar la imagen del cosmos constituida de esferas cristalinas concéntricas con las que se encontraba estructurado el 
cosmos tardomedieval. Pero, por una parte, esto no era condición suficiente para la disolución de la esfera celeste y, por otra parte -y mucho más importante-, la disolución de la esfera celeste no era condición necesaria para el paso dado por Galileo. El paso dado por Galileo fue hacia la concepción de un horizonte como un círculo necesariamente máximo en una esfera considerada como proyección. Pero concebir a la esfera como proyección no supone la disolución de una última esfera.

El cambio de Galileo no podría explicarse entonces ni por un conjunto de nuevas observaciones celestes puntuales ni por el hecho de que un cambio teórico colisionara con un acervo de observaciones preexistentes. De allí, la necesidad de explorar más allá de las estrictas fronteras de la teoría y la práctica astronómicas. En lo que sigue, analizaré la posibilidad de que Galileo haya integrado en su interpretación de la experiencia visual el conocimiento producido y desarrollado con anterioridad en el ámbito de las artes mecánicas como producto de las investigaciones del espacio desarrolladas a propósito de la representación pictórica en perspectiva. Para ello, en el próximo apartado consideraré algunas características relevantes de tal conocimiento.

\section{La perspectiva y la esfera celeste de Galileo}

U no de los temas centrales del arte renacentista italiano fue el establecimiento de un nuevo diálogo entre apariencias perceptivas y realidades espaciales. Esto se manifestó de manera elocuente en la pintura, ámbito en el que un objetivo primordial fue el desarrollo de nuevas técnicas y métodos para el estudio y la representación en dos dimensiones del espacio tridimensional. El desiderátum de la transformación era alcanzar una representación lo más perfecta posible de la realidad, perfección que o bien no era buscada o bien era concebida de manera distinta en el arte medieval y en otras partes de Europa en ese mismo período.

En la pintura, una de las manifestaciones más claras del cambio en la relación apariencia-realidad fue el desplazamiento de las jerarquías intrínsecas de lo pintado; la lógica de acuerdo con la cual la relevancia del objeto era la ordenadora del espacio fue reemplazada por jerarquías cuantificadas en función de la distancia respecto del observador. El lugar del sujeto que observaría la representación artística pasó a ser determinante en la constitución de la obra, resignificando los papeles del sujeto y el objeto artísticos. En dicho contexto, uno de los elementos que los artistas debieron considerar fue la forma en la que el espacio tridimensional debía proyectarse sobre las dos dimensiones de la representación de manera tal 
que el espacio representado adquiriera coherencia para el observador. En un breve período de tiempo tuvieron lugar procesos mentales que condujeron a elaborar condiciones para abstraer y representar lo real de manera novedosa. Al igual que en otras disciplinas del período, un paso fundamental en ese cambio fue la introducción sistemática del uso de la geometría; ella se volvió una condición de posibilidad para la representación de lo observado, lo que era consistente con la creciente convicción de que la naturaleza poseía tales cualidades geométricas.

En general, existe un acuerdo en que la introducción de la geometría en las artes mecánicas tuvo un impacto mayúsculo en su contenido, su práctica y su caracterización. Esto se manifestó, por ejemplo, en la eliminación de cierta barrera que las separaba de las artes liberales (hoy referidas como ciencias, reunidas en el trivium y el quadrivium), proceso que se dio especialmente con la pintura y la arquitectura. Uno de los resultados de las investigaciones llevadas a cabo por los artistas de la época es lo que hoy se conoce con el nombre de perspectiva, conocimiento diferente de aquel conocido con el mismo nombre en el período medieval y al que hoy referimos generalmente como óptica (véase Lindberg 2002). Mientras que este último se orientaba al estudio de la naturaleza de la luz -o bien desde una perspectiva metafisica o bien como una ciencia empírica-, la perspectiva desarrollada en el ámbito artístico moderno fue una técnica pictórica diseñada y aplicada sistemáticamente desde el Quattrocento de manera consciente y novedosa.

Posiblemente, la primera ejecución práctica del conjunto de técnicas que luego catalizarían en la perspectiva fue la construcción por parte de Filippo Brunelleschi (1377-1446) de una pintura del Battistero di San Giovanni (en Florencia) que, observada bajo determinadas condiciones, generaba al observador la ilusión de estar viendo el propio edificio. Lo que demostraba de manera práctica el experimento de Brunelleschi era la posibilidad de generar una impresión de un espacio tridimensional sobre una superficie bidimensional. Las claves para que ello funcionara eran que se respetaran la posición y las distancias entre la pintura y el observador y que el observador permaneciera quieto observando con un solo ojo a través del orificio diseñado para tal fin (para evitar que la visión estereoscópica arruinara el efecto) y, desde luego, que la pintura estuviera compuesta de una determinada forma.

Es de suponer que esa forma no era muy distinta de la que tiempo más tarde sistematizaría y pondría por escrito León Battista Alberti (14041472), en su tratado Della Pittura, de 1453. Alberti dice que la técnica de representación geométrica con proyección central se construye sobre la base de concebir el acto de la observación como una pirámide, definida como: 
cuerpo oblongo de cuyas bases surgen líneas rectas que prolongadas hacia arriba terminan en un punto común. La base de la pirámide es la superficie observada y los lados son los rayos visuales que denominamos extrínsecos. La cúspide [vértice] de la pirámide se encuentra dentro del ojo donde los ángulos de las cantidades en los distintos triángulos se unen [...]; de todo ello resulta evidente que la distancia entre la superficie y el ojo posee una importancia considerable. (Alberti 1996: 72-73)

La idea rectora es que la forma que asume lo representado depende del sujeto de la observación y de la ubicación del plano sobre el que se proyecta la imagen observada. Para tal fin, la sugerencia albertiana era utilizar un velo que, interpuesto entre la imagen a pintar y el observador, permitiera a este último encontrar la cúspide de la pirámide visual y establecer los límites.

Las reglas básicas a seguir para la reconstrucción geométrica del espacio de acuerdo con esta forma de perspectiva son: a) que las líneas rectas sean, en la representación, siempre líneas rectas (excepto la que pasa por el centro de la proyección, que asume la forma de punto); b) que las líneas paralelas al plano de la pintura sean, en la representación, paralelas entre sí; y c) que las líneas que no son paralelas al plano de representación converjan entre sí en uno o más puntos, denominados puntos de fuga. Dichos puntos de fuga se definen tanto vertical como horizontalmente en función de la posición de la pintura en un determinado espacio físico y, consecuentemente, la posición que el observador tendrá respecto de ella.Verticalmente, a partir del ángulo de la visual y de la pretensión de orientación de la pintura. Horizontalmente, configurado a partir de una línea a la altura de los ojos del observador, que constituye la línea del horizonte.

Los requisitos señalados son todos igualmente relevantes al momento de lograr el efecto de la profundidad de la pintura de acuerdo con esta forma de perspectiva. Y es especialmente importante que los puntos de fuga se establezcan considerando la proyección de la visual del observador, por lo que deben considerar la ubicación que adoptará el observador respecto de la pintura. Si, por ejemplo, se cumplieran las reglas $a-c$, pero luego se estableciera un punto de fuga inconsistente con la posición de observador, entonces la representación no alcanzaría su objetivo. En este sentido, es de destacar que el mayor objeto de investigación, dados esos requisitos, era cómo establecer con precisión el punto (o los puntos) de fuga con relación a la posición que debería tomar el observador para que este vivenciara la ilusión de fondo (al mirar, cabe reiterar, con un solo ojo inmóvil).

Exceden los propósitos de este trabajo los pormenores técnicos de los estudios que tuvieron lugar en el período renacentista sobre las formas más convenientes de representar el espacio, como los intentos de construir 
una perspectiva sintética (o naturalis) que, por oposición a la regla a de la perspectiva artificialis referida hasta aquí, curva las líneas rectas en proporciones dependientes de las distancias al plano del horizonte (al respecto, véase White 1987: 287 ss.). Alcanza con señalar que supuso un estudio profundo de la relación entre el horizonte y el observador, que es una de las formas de la indagación en torno a la interacción entre el observador y lo observado y la representación y lo representado ${ }^{2}$.

La relevancia del tema se manifestó no solo en textos sino también en imágenes, como la serie de grabados de Albrecht Dürer incorporados en distintas ediciones de su obra Underweysung der Messung en los que se retratan distintas técnicas para la composición de una representación con proyección central. Especialmente elocuente es la reproducida en la Imagen 1 incorporada en la tercera edición de 1538. En ella, vemos un horizonte geográfico representado en el fondo de la imagen que es no solo el horizonte perspectivo de la propia imagen sino también, y como se puede adivinar por la posición del ojo del dibujante y por el velo es también la línea del horizonte de lo que será su producción; su instrumento de dibujo aparece no casualmente posado sobre la línea del horizonte en la que en encuentra el punto de fuga de su futuro dibujo.

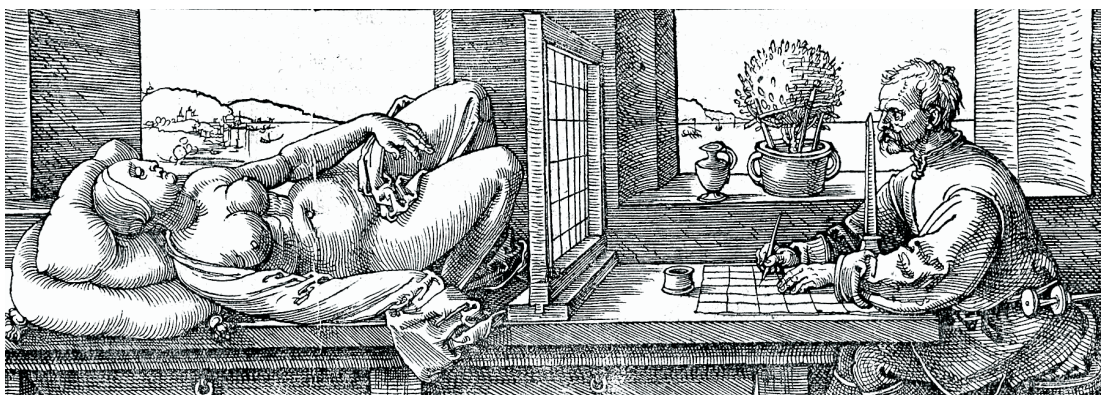

Imagen 1

Artista pintando un desnudo, xilografia presente en la tercera edición de Underweysung der Messung de Albrecht Dürer, de 1538. Imagen de dominio público.

Los estudios sobre la forma en la que el espacio se proyecta sobre un plano de representación favorecieron el desarrollo de una idea proyectiva. El logro de la representación en perspectiva artificialis fue superar un viejo escollo enunciado en el octavo postulado de la Óptica de Euclides, que establecía

\footnotetext{
${ }^{2}$ Véase Panofsky 2003, Jones y Hagen 1978 y Schröder 1980.
} 
que "magnitudes iguales y paralelas, desigualmente distantes del ojo, no son vistas con proporcionalidad a las distancias respecto del ojo" (Euclides 2000a: 142). A modo de ejemplo, en la representación de la izquierda en la Figura 2 los segmentos $A B$ y $C D$ son congruentes, y la distancia al observador $O$ del primero es la mitad que la del segundo. Esa proporcionalidad en la distancia no se mantiene en el ángulo que subtienden en el observador, ya que . En la representación de la derecha en la misma figura, se puede apreciar que el plano proyectivo que contiene a los puntos $E, F$ y $G$ y que corta la pirámide visual construye triángulos $O E F$ y $O E G$ que son proporcionales a los triángulos $O A B$ y $O C D$, respectivamente (para una explicación detallada, véase Burucúa 1991). Así, la geometría se constituía en un elemento central para la solución de problemas prácticos en el ámbito de las artes mecánicas.

Postulado euclídeo

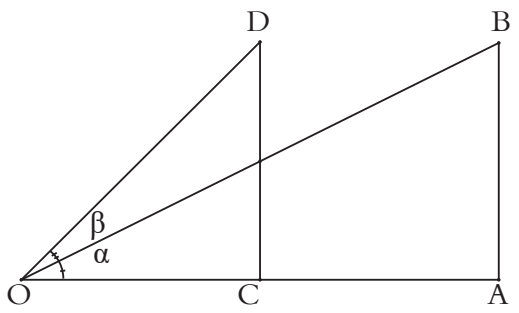

Proporcionalidad proyectiva

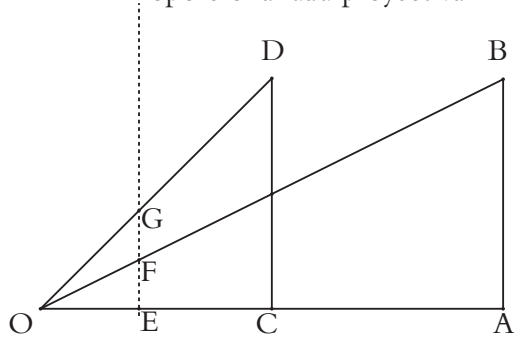

Figura 2

Comparación del postulado euclíedo de la no proporcionalidad de los ángulos subtendidos por dos magnitudes iguales con las distancias al observador, y la proporcionalidad de los triángulos contruidos sobre la superficie proyectiva de acuerdo con la perspectiva artificialis. Figura original del autor.

Se señaló más arriba que la introducción de la geometría modificó el carácter de las artes mecánicas y su relación con las artes liberales. Al respecto, no parecen existir disensos. Tampoco hay disenso sobre el hecho de que a esa disolución también contribuyó la creciente aspiración de las ciencias de investigar la naturaleza mediante el uso de la experiencia. Sobre lo que sí existió y existe en alguna medida disenso es en torno a la relevancia que los cambios producidos en el marco del desarrollo artístico tuvieron en el desarrollo de las ciencias durante la Revolución Científica. Considerar los posibles vínculos entre el cambio astronómico presentado en la sección anterior y el cambio en la percepción espacial renacentista, permitirá echar luz sobre este problema.

En la reinterpretación galileana de la experiencia considerada en el apartado anterior, la totalidad del fenómeno visible se relativizó al 
observador, siendo consecuencia de una realidad muy distinta de la concebida por Ptolomeo. Su respuesta mostró que quienes esgrimían el comportamiento de los astros sobre el horizonte para probar la posición central de la Tierra suponían que esta se encontraba en reposo y, por tanto, el horizonte también. Al suponer eso, el cielo y sus partes, así como sus movimientos, eran considerados absolutos y, dados los fenómenos, permitían inferir la posición central del observador y, consecuentemente, de la Tierra. Abandonado tal supuesto, no había necesidad de continuar concibiendo, describiendo o interpretando al cielo y a sus partes de ese modo (aunque los primeros copernicanos no lo hubieran advertido).

En cierto sentido, Galileo observó la misma esfera que observaron Ptolomeo o Copérnico, pero, en otro sentido, no: los conceptos utilizados por unos y otro para interpretarla eran tan distintos que, posiblemente, la propia observación fuera distinta. Entre ellos, de capital relevancia fueron los conceptos de horizonte y de esfera. Mientras para Ptolomeo el horizonte era un círculo de la esfera accidentalmente máximo por estar la Tierra en su centro, el horizonte galileano era un círculo máximo necesariamente independiente de la posición de la Tierra en el universo, por cuanto resultaba de la proyección de la visual de un observador sobre una esfera aparente con radio indeterminado y con centro en su ojo.

Tal reinterpretación se derivaba de la descomposición de una única esfera celeste en dos (o, para ser precisos, en algo de lo que no podía saberse ni su forma ni su tamaño y en una esfera). Con ello, la imagen del cielo fue descompuesta en dos: algo real, el universo, y algo aparente, una esfera que no era más que proyección radial de ese universo real. Galileo hizo evidente que somos el centro necesario de lo aparente y que no podemos saber si estamos en el centro de lo real. Los observadores terrestres estamos en el centro de la eclíptica, del ecuador y en una posición equidistante de los polos, pero ellos no son más que la proyección de puntos y líneas descritos por movimientos relativos de la Tierra sobre esa esfera proyectiva centrada necesariamente en nosotros.

Esta posibilidad de descomponer entre lo real y lo proyectado se encuentra en sintonía con la capacidad de repensar lo observado bajo las premisas de una idea proyectiva desarrollada a instancias de la perspectiva en el ámbito de la pintura. Entender que una proyección puede parecerle real al ojo del observador siempre que en la superficie proyectada adquiera una imagen geométricamente consistente es la clave de la perspectiva. Lo es, también, de la reinterpretación galileana de la observación astronómica del universo. Y es un rasgo característico de la observación astronómica el que permite establecer un puente entre la reconstrucción de la espacialidad de fondo de la pintura y la percepción psicofisiológica que tiene lugar cuando 
el horizonte delimita una ventana al cielo: mientras que en la observación del cuadro en perspectiva es necesario que el observador permanezca en reposo observando con un solo ojo (para evitar que la visión estereoscópica devele el engaño), el observador astronómico terrestre está condenado a observar de ese modo. Sea que mire con un ojo o con los dos, sea que no se desplace en el espacio o que lo haga una cantidad despreciable como lo es la distancia que lo separa del sol, a los fines prácticos, es como si el observador celeste hubiera estado observando al universo siempre con un solo ojo y desde el mismo lugar.

La ausencia de una referencia explícita por parte de Galileo a la posibilidad de este parangón, hace que cualquier interpretación sobre la relevancia del contenido de las investigaciones en las artes figurativas como condición de posibilidad para su nueva aproximación a un problema cosmológico-astronómico sea altamente especulativa. Pero tal especulación se encuentra guiada por una multiplicidad de estudios sobre el vínculo de Galileo con las artes figurativas que contribuyeron a responder afirmativamente la elocuente pregunta de Panonfsky "si se considera que la actitud científica de Galileo ha influido en su juicio estético, ¿no podría su actitud estética haber influido en sus teorías científicas?” (Panofsky 1956: 11).

Un ejemplo categórico es el reseñado por Edgerton ${ }^{3}$, quien muestra comparativamente cómo Galileo fue capaz de comprender las irregularidades de la Luna como manifestación de distintas profundidades en su superficie de manera distinta a como lo hizo Thomas Harriot (1560-1621). En la Imagen 2, los dibujos (a) y (d) son de Harriot y los dibujos (b) y (c) son de Galileo. Mientras que el dibujo (a) de Harriot es anterior al de Galileo, el dibujo (d) es posterior. Respecto de lo dibujado en (a), no pueden aducirse exclusivamente dificultades técnicas (ni de dibujo ni de observación), puesto que el mismo Harriot, ya influenciado por Galileo, fue capaz de dibujar algo bastante más próximo a una idea de profundidad, en la imagen (d). Con lo que, evidentemente, la sensibilidad original para captar el espacio de uno y otro observador era distinta, aunque esto no impidió a Harriot aprender a visualizar lo que veía Galileo una vez que fue orientado hacia tal sensibilidad. Pumfrey (2009) buscó establecer que los dibujos de Harriot tenían tales características porque su objetivo era calcular la libración lunar y que por ello es incorrecto compararlos con los de Galileo, que perseguían otros fines. Si bien parece pertinente la idea de no considerar a unos dibujos superiores respecto de los otros, su señalamiento no cambia el hecho de que los dibujos

\footnotetext{
${ }^{3}$ Véase Edgerton 1984 y 1991.
} 
muestran una sensibilidad distinta al espacio; aun buscando establecer la libración, no había motivos para que dejara de representar las profundidades como tales, si acaso era capaz de percibirlas.

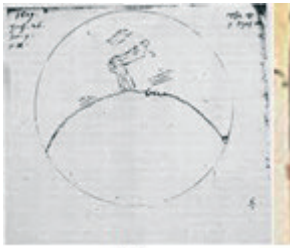

(a)

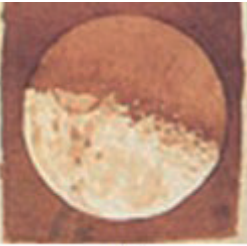

(b)

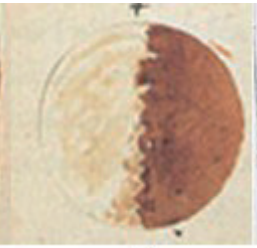

(c)

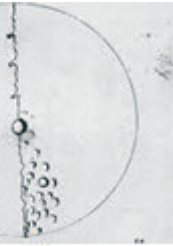

(d)

\section{Imagen 2}

Comparación de los dibujos de la luna hechos por Galileo (b y c) y Harriot (a y d) de acuerdo con la investigación de Edgerton. Imágenes en Edgerton (1984: 227-228).

Sobre la originalidad de las aspiraciones de Galileo de trazar representaciones naturales del cielo, en contraposición con una tradición casi sin excepciones preocupada exclusivamente por integrar diagramas, se expiden Winkler y Van Halden (1992), quienes no dudan en señalar como decisiva la influencia de la cultura renacentista, de las artes mecánicas en general y de la pintura en particular, en la aproximación de Galileo a la naturaleza (en contraposición con otros filósofos naturales de la época). Más cerca en el tiempo, los estudios conducidos por Bredekamp, incluidos aquellos fomentados por la investigación de una primera edición apócrifa del Sidereus Nuncius, arrojaron luz sobre la relevancia capital del conocimiento artístico en la interpretación y representación de la naturaleza por parte de Galileo. ${ }^{4}$

Pero el estudio más íntimamente vinculado al tema tratado aquí es anterior. En su tesis doctoral, Burucúa estableció de manera extensamente documentada la existencia de una idea proyectiva en Galileo (Burucúa 1984). De acuerdo con esa investigación, si bien la biblioteca de Galileo no contaba con volúmenes sobre perspectiva, pueden señalarse sobrados elementos que, conjuntamente con los dibujos, permiten establecer su dominio del tema: a) contacto con artistas a lo largo de su vida, b) referencias a artistas en sus escritos, c) reflexiones explícitas al arte en el marco de discusiones filosóficas, d) uso de un lenguaje que lo muestra familiarizado con los desarrollos de la perspectiva del momento, y e) instrumentos para la observación celeste que es muy plausible que fueran adaptaciones de instrumentos propios del ámbito

\footnotetext{
${ }^{4}$ Véase Bredekamp, Brückle, Hahn, y Needham 2011; Bredekamp 2012 y 2015.
} 
artístico. Todo esto da un contexto a la especulación sobre la influencia de las artes figurativas en la respuesta galileana al problema astronómico que refuerza el mero vinculo temático o conceptual entre uno y otro.

Por otra parte, la posibilidad de repensar lo indiscernible de la posición en el universo dadas las apariencias celestes no fue algo que se dio exclusivamente a partir de la intervención de Galileo en los debates astronómicos. Con anterioridad a él, al menos dos filósofos de talla exploraron las apariencias celestes para observadores en universos infinitos y llegaron a una conclusión semejante sobre la necesidad de diferenciar la apariencia de que el universo se encuentra centrado en uno con la realidad de que así sea. Me refiero a Nicolás de Cusa y a Giordano Bruno, quienes postularon universos infinitos por motivos metafísicos y sostuvieron que las apariencias eran consistentes con su observación desde un punto de vista no central (véase Levinas y Szapiro 2013). Estos antecedentes, lejos de desviar nuestra atención de las formas en las que el conocimiento artístico pudo haber influido sobre los procesos de descentración cosmológica, nos persuaden de su importancia. Porque distintos estudios han constatado, para tales antecesores, similares influencias.

Sobre Bruno, ya Panofsky señaló un fuerte paralelismo entre los cambios que estaban teniendo lugar en el ámbito artístico y en el pensamiento abstracto:

En los mismos años en que la espacialidad del Giotto y del Duccio, análoga a la concepción en transición de la alta escolástica, estaba siendo superada mediante la gradual elaboración de la verdadera perspectiva central con su espacio ilimitadamente extenso y organizado en torno a un punto de vista elegido a voluntad, el pensamiento abstracto llevaba a cabo de un modo abierto la ruptura, anteriormente velada, con la visión aristotélica del mundo, renunciando a la concepción de un cosmos construido en torno a centro de la Tierra, es decir, en torno a un centro absoluto y rigurosamente circunscrito por la última esfera celeste, desarrollando así el concepto de una infinitud, no solo prefigurada en Dios, sino realizada de hecho en la realidad empírica [...] no es de sorprender que un hombre como Giordano Bruno dotara de una sublimidad casi religiosa este mundo de la espacialidad infinita. (Panofsky 2003: 47).

Mucho más cerca en el tiempo, Ordine (2008) realizó un completo estudio sobre los vínculos del Nolano con las artes figurativas y la literatura de la época, enfatizando el valor de la pintura como metáfora en la obra bruniana. Pero quizá aun más pertinente a este respecto es el estudio realizado porVidal y Burucúa, en el que se concluye que hacia la composición 
del De Inmenso, Bruno había abandonado la convicción de "la insuficiencia demostrativa de la visión matemática, certeza que le ocluyó en principio la posibilidad de pensar el punctum concursus [punto de fuga] como 'representación' visual del infinito" y había "integrado los datos de la visibilidad y la representación perspectiva entre los elementos probatorios más poderosos de la infinitud del universo" (Vidal y Burucúa 2004: 146).

También en el pensamiento de Nicolás de Cusa parece haber sido decisiva la escena que estaba teniendo lugar en Italia, con la que tomó contacto inicial entre 1417 y 1423 al estudiar derecho y matemáticas en Padua (donde recibió formación de parte de eminentes figuras de la época como Pablo Toscanelli) y con la que parece haber continuado en contacto por el resto de su vida. Hopkins se atreve a señalar que: "dentro del sincretismo de la teoría de Nicolás de Cusa, debe dársele mayor prominencia a una figura histórica: León Battista Alberti, por cuyas obras De Pictura, Elementa Picturae y De Ludi Mamematici Nicolás fue aparentemente influenciado" (Hopkins 1996: 51-2). Si bien Hopkins se refiere sobre todo a la influencia que puede ser detectada en obras posteriores a aquella en la que el Cusano postula un universo infinito, De Docta Ignorantia, cronológicamente es perfectamente posible que esta influencia hubiera tenido lugar ya en ella. Riedenauer parece ir en esa dirección al señalar que se puede establecer un parangón entre la epistemología del Cusano y la perspectiva linear, y que hacia la época de $D e$ Docta Ignorantia ya había comenzado la transferencia del "fenómeno de la vista natural a la vista de la mente" (Riedenauer 2005: 284). Señala que, más tarde, la obra del Cusano presentaría todavía un mayor grado de síntesis con la de Alberti, en el mismo sentido que lo había anticipado Santinello (1958) y que más recientemente continuó estudiando Carman. ${ }^{5}$

En el punto específico en consideración Galileo no es, conviene aclarar, un simple heredero del pensamiento del Cusano o del Nolano. Estos últimos postulaban un universo infinito como consecuencia de sus consideraciones metafisicas e integraban los datos sensibles desde esa presunción. Galileo, en el contexto específico de esta carta a Ingoli, toma por el contrario distancia de la posibilidad de establecer un conocimiento al respecto: "y no sabes tú que es todavía indeciso (y creo que será siempre entre las ciencias humanas) si el universo es finito o infinito?" (Galilei 1933b: 529). Así, la compatibilidad con la experiencia no requiere en él de la presunción de un espacio infinito sino simplemente de la constricción del observador a un punto de observación. En este sentido, la especulación galileana es esencial-

\footnotetext{
${ }^{5}$ Véase Carman 2007 y 2014.
} 
mente original. Esa especial combinación entre, por una parte, la familiaridad de las consideraciones cosmológicas de distintos filósofos naturales interesados por las artes figurativas y, por otra, sus particulares diferencias, lejos de socavar la tesis de una influencia de las artes mecánicas sobre la ciencia, parece sugerir que tal influencia existió de diversas formas que manifiestan cierto grado de similitud.

Desde un punto de vista histórico resulta elocuente que los tres primeros exponentes de un pensamiento que compatibilizó (de distintas formas) la experiencia observacional con la descentración del observador hayan tenido un interés por los desarrollos técnicos en el ámbito de las artes figurativas. Desde un punto de vista epistemológico, no deja de llamar la atención que ninguno de los tres pueda ser considerado, en sentido estricto, astrónomo de formación o de profesión. Quizá ambas condiciones hayan cooperado para que pudieran quitarse de encima el yugo conceptual ptolemaico.

\section{Conclusiones}

$\mathrm{n}$ el curso de este trabajo, presenté la novedad y especificidad de la aproximación de Galileo Galilei a un antiguo debate astronómico con consecuencias cosmológicas. Señalé que las reflexiones sobre las apariencias del espacio de tal aproximación manifiestan un grado de familiaridad con las ideas del espacio desarrollados en el ámbito pictórico a propósito del desarrollo de la perspectiva, por las que Galileo tuvo interés y las que, de acuerdo con diversas investigaciones, influyeron en su interpretación y representación de la naturaleza en problemas distintos del considerado aquí. Consideré también que la aproximación de Galileo al problema cosmológico específico tiene paralelismos con las ideas de otros filósofos naturales que, antes que él, desafiaron la capacidad probatoria de las apariencias celestes para determinar una posición central del observador en el universo y que, al igual que él, tenían conocimiento de, e interés por, la perspectiva. De conjunto, la investigación dio sustento a la idea de que la operación cognitiva que hizo Galileo, expresada en su texto de 1624, volvió a combinar y entretejer los desarrollos lógico-matemáticos y los fenómenos desmenuzados por la observación, cuya verdad y fertilidad gnoseológica la perspectiva había desvelado.

Lo considerado aquí me conduce, inevitablemente, a tomar parte en el debate sobre la influencia de las artes mecánicas en el desarrollo científico del siglo XVII a favor de tesis como la de Burucúa, quien sostiene que "las artes figurativas fueron uno de los pilares más sólidos en los que se apoyó la 
Revolución Científica" (Burucúa 1984: 18) y en contra de posiciones como las de Stephen Toulmin, quien afirma que "por más diferentes que hubieran sido la poesía y el drama, la música y la arquitectura del siglo XVII, la revolución cosmológica hubiera ocurrido igual, en todos sus aspectos esenciales, exactamente como lo hizo" (Toulmin 1961:11).

Las reflexiones sobre el espacio desarrolladas en el ámbito pictórico sin dudas no agotan las fuentes de inspiración y conocimiento que permitieron el cambio reseñado en la primera sección de este trabajo; indudablemente conocimientos resultantes de otros fenómenos, como la expansión ultramarina, pudieron funcionar también como fundamento de las especulaciones sobre la espacialidad, su percepción y su representación aquí consideradas. La evaluación de tales vínculos excede los propósitos de este trabajo; la constatación de su existencia confirmaría, por una parte, la dependencia de los cambios conceptuales analizados aquí de procesos que tuvieron lugar fuera del ámbito estrictamente astronómico-cosmológico y, por otra, la relevancia metodológica de la indagación transdisciplinar y de la consideración de factores extracientíficos para la comprensión de la historia del conocimiento científico.

\section{BIBLIOGRAFÍA}

Alberti, L. B. (1996), De la pintura (México: UNAM).

Aristóteles y Euclides (2000), Sobre las líneas indivisibles - Mecánica - Óptica - Catóptrica Fenómenos (Madrid: Gredos).

Bredekamp, H. (2012), Galilei der Künstler: Der Mond. Die Sonne. Die Hand (Berlin: Walter de Gruyter).

Bredekamp, H. (2015), Galileis denkende Hand: Form und Forschung um 1600 (Berlin: Walter de Gruyter).

Bredekamp, H., Brückle, I., Hahn, O y Needham, P. (2011), Galileo's O. (Berlin: Akademie Verlag).

Burucúa, J. E. (1984), El libro de la naturaleza: estudio acerca de las ideas de Galileo Galilei sobre las artes figurativas. Tesis de Doctorado (Buenos Aires: Universidad de Buenos Aires).

Burucúa, J. E. (1991), “Nugae perspectivae: el debate sobre la percepción del espacio tridimensional y su representación”, Temas medievales, 1:39-80.

Carman, C. H. (2007), "Alberti and Nicholas of Cusa: Perspective as 'Coincidence of Opposites"”, Explorations in Renaissance Culture, 33: 196-219.

Carman, C. H. (2014), Leon Battista Alberti and Nicholas Cusanus:Towards an Epistemology of Vision for Italian Renaissance Art and Culture (Burlington: Ashgate).

Copérnico, N. (1997), Sobre las revoluciones (de los orbes celestes) (Barcelona:Altaya). 
Copérnico, N., Digges, T. y Galilei, G. (1983), Opúsculos sobre el movimiento de la Tierra (Madrid:Alianza).

Digges, T. (1983), "Una perfecta descripción de las esferas celestes según la antiquísima doctrina de los pitagóricos, recientemente revivida por Copérnico y acreditada por medio de demostraciones geométricas”, en N. Copérnico, T. Digges y G. Galilei (1983), Opúsculos sobre el movimiento de la Tierra (Madrid: Alianza, 45-63).

Edgerton, S.Y. (1984), “Galileo, Florentine 'Disegno', and the 'Strange Spottednesse' of the Moon”, The Art Journal / College Art Association of America, 44: 225-232.

Edgerton, S.Y. (1991), The Heritage of Giotto's Geometry: Art and Science on the Eve of the Scientific Revolution (Ithaca: Cornell University Press).

Euclides (2000a), “Óptica”, en Aristóteles y Euclides (2000), Sobre las líneas indivisibles Mecánica - Óptica - Catóptrica - Fenómenos (Madrid: Gredos, 117-197).

Euclides (2000b), "Fenómenos", en Aristóteles y Euclides (2000), Sobre las líneas indivisibles - Mecánica - Óptica - Catóptrica - Fenómenos (Madrid: Gredos, 241-324).

Favaro, A. (1891) (ed.), Le opere di Galileo Galilei: edizione nazionale sotto gli auspicii di Sua Maestà il Re d'Italia, Vol II (Firenze: Tipografia di G. Barbèra).

Galilei, G. (1891), “Tratatto della sfera ovvero Cosmografia” en Favaro (1891) (ed.), Le opere di Galileo Galilei: edizione nazionale sotto gli auspicii di Sua Maestà il Re d'Italia, Vol II (Firenze: Tipografia di G. Barbèra, 211-255).

Galilei, G. (1933a), Le opere di Galileo Galilei (Firenze: G. Barbera).

Galilei, G. (1933b), "Lettera a Francesco Ingoli”, en Galilei (1933b), Le opere di Galileo Galilei (Firenze: G. Barbera, 6: 509-561).

Hopkins, J. (1996), Nicholas of Cusa on Wisdom and Knowledge (Minneapolis: A. J. Banning Press).

Johnson, F. R. y Larkey, S. V. (1934), "Thomas Digges, the Copernican System, and the Idea of the Infinity of the Universe in 1576", The Huntington Library Bulletin, 5: 69-117.

Jones, R. K. y Hagen, M. A. (1978), “The Perceptual Constraints on Choosing a Pictorial Station Point", Leonardo 11:191-196.

Koyré, A. (1979), Del mundo cerrado al universo infinito (México: Siglo XXI).

Levinas, M. L. y Szapiro A. (2013), "La noción de horizonte como reflejo de las disputas astronómicas en torno a la posición de la Tierra (1440-1624)”, Scientiae Studia - Revista Latino-Americana de Filosofia e História da Ciência, 11: 763-784.

Lindberg, D. C. (2002), Los inicios de la ciencia occidental. La tradición científica europea en el contexto filosófico, religioso e institucional: desde el 600 a. C. hasta 1450 (Barcelona: Paidós).

Machetta, J. M. y D’Amico, C. (2005) (eds.), El problema del conocimiento en Nicolás de Cusa: genealogía y proyección (Buenos Aires: Biblos).

Ordine, N. (2008), El umbral de la sombra: literatura, filosofía y pintura en Giordano Bruno (Madrid: Siruela). 
Panofsky, E. (1956), "Galileo as a Critic of the Arts: Aesthetic Attitude and Scientific Thought", Isis, 47: 3-15.

Panofsky, E. (2003), La perspectiva como "forma simbólica" (Barcelona:Tusquets).

Ptolomeo, C. (1984), Ptolemy's Almagest (New York: Springer).

Pumfrey, S. (2009), "Harriot's Maps of the Moon: New Interpretations", Notes and Records of the Royal Society, 63: 163-168.

Rhys, H. H. (1961) (ed.), Seventeenth Century Science and the Arts (Princeton: Princeton University Press).

Riedenauer, M. (2005), "Pluralità di prospettive finite nell'orizzonte dell'infinito: Conseguenze della epistemologia nuova di Cusano” en J. M. Machetta y C. D’Amico (2005) (eds.), El problema del conocimiento en Nicolás de Cusa: genealogía y proyección (Buenos Aires: Biblos, 281-290).

Santinello, G. (1958), Il pensiero di Nicolò Cusano nella sua prospettiva estetica (Padova: Liviana)

Schröder, E. (1980), Dürer Kunst und Geometrie: Dürers künstlerisches Schaffen aus der Sicht seiner Underweysung (Basel-Boston-Stuttgart: Birkhäuser).

Szapiro, A. (2018), “Assumptions behind Ptolemy's Proof of the Earth's Centrality”, Journal for the History of Astronomy, 49: 196-215.

Toulmin, S. (1961), "Seventeenth Century Science and the Arts", en H. H. Rhys (1961) (ed.), Seventeenth Century Science and the Arts (Princeton: Princeton University Press, 3-28).

Vidal, S. P. y J. E. Burucúa (2004), "La perspectiva naturalis y la perspectiva artificialis en la obra de Giordano Bruno: los avatares de su dialéctica entre los argumentos a favor de la infinitud del universo", Revista Latinoamericana de Filosofía, 30: 125-146.

White, J. (1987), The Birth and Rebirth of Pictorial Space (London: Faber and Faber).

Winkler, M. G. y Helden, A.V. (1992), "Representing the Heavens: Galileo andVisual Astronomy", Isis, 83: 195-217.

Recibido: 06-03-2019; aceptado: 23-07-2019 